\title{
PERFECCIONISMO Y DESEMPEÑO ACADÉMICO EN ESTUDIANTES UNIVERSITARIOS DE LA CIUDAD AUTÓNOMA DE BUENOS AIRES ${ }^{\perp}$
}

\author{
FERNÁN GUIDO ARANA*, MARINA SOLEDAD GALARREGUI, MARIANA CAROLINA MIRACCO \\ CONSEJO NACIONAL DE INVESTIGACIONES CIENTÍFICAS Y TÉCNICAS (CONICET) \\ INSTITUTO DE INVESTIGACIONES \\ FACULTAD DE PSICOLOGÍA \\ UNIVERSIDAD DE BUENOS AIRES - ARGENTINA \\ ANDRÉS IGNACIO PARTARRIEU, LORENA DE ROSA, ADRIANA ELBA LAGO, LILIANA ISABEL TRAIBER, \\ PATRICIO TOMÁS NUSSHOLD, GUILLERMINA RUTSZTEIN, EDUARDO GUSTAVO KEEGAN \\ INSTITUTO DE INVESTIGACIONES \\ FACULTAD DE PSICOLOGÍA \\ UNIVERSIDAD DE BUENOS AIRES - ARGENTINA
}

Recibido, junio 2/2013

Concepto evaluación, noviembre 21/2013

Aceptado, diciembre 20/2013
Referencia: Arana, F. G., Galarregui, M. S., Miracco, M. C., Partarrieu, A. I., De Rosa, L., Lago, A. E., Traiber, L. I., Nusshold, P. T., Rutsztein, G. \& Keegan, E. G. (2014). Perfeccionismo y desempeño académico en estudiantes universitarios de la Ciudad Autónoma de Buenos Aires. Acta Colombiana de Psicología, 17(1), 71-77. doi: 10.14718/ACP.2014.17.1.8

Resumen

El presente artículo tiene por objetivo analizar la relación entre los perfiles de perfeccionismo y el desempeño académico en una muestra de estudiantes universitarios de la Ciudad de Buenos Aires. El desempeño ha sido operacionalizado a través de las variables promedio académico general percibido, calificación del último examen, cantidad de exámenes adeudados $y$ años de carrera cursados. Participaron en la investigación 465 estudiantes pertenecientes a las carreras de Psicología $(52,2 \%)$ Ingeniería $(26,2 \%)$ y Medicina $(21,6 \%)$. La distribución de la población de estudiantes en los distintos perfiles de perfeccionismo (adaptativo- PA, desadaptativo- PD y no perfeccionistas-NP) se presentó según las predicciones derivadas de la teoría y los estudios previos realizados por nuestro equipo de investigación. Respecto a la relación entre los diferentes perfiles y el desempeño académico, se encontró que los estudiantes con un perfil de PA presentaron un promedio percibido significativamente superior, y una cantidad de exámenes adeudados significativamente menor que los estudiantes de los restantes perfiles. No se evidenciaron diferencias significativas para estas variables entre los sujetos con un perfil de PD y los estudiantes NP. Para las restantes variables no se hallaron diferencias significativas entre los distintos perfiles de perfeccionismo. Se discuten las implicancias de estos hallazgos para futuras investigaciones en el área.

Palabras Clave: perfeccionismo - desempeño académico - estudiantes universitarios

\section{PERFECTIONISM AND ACADEMIC PERFORMANCE OF UNIVERSITY STUDENTS IN THE AUTONOMOUS CITY OF BUENOS AIRES}

\begin{abstract}
The aim of this article is to analyze the relationship between perfectionism and academic performance of university graduate students in the City of Buenos Aires. 465 university undergraduates participated in this study: Psychology students (52.2\%), Engineering students (26.2\%) and Medicine students (21.6\%). The resulting scores used to set the different perfectionism profiles (adaptative perfectionists- AP, maladaptive perfectionists- MP, and non perfectionists- NP), are similar to those expected according to current theory and to our previous studies. Regarding the relationship between the different student
\end{abstract}

\footnotetext{
* Bahía Blanca 1838, departamento 6, C1407ACJ. Ciudad Autónoma de Buenos Aires, República Argentina. Número telefónico 5411 2050 2816. fernanarana@gmail.com, Correo electrónico de los demás Autores en su orden: mgalarregui@psi.uba.ar, mmiracco@psi.uba.ar, androspsi@yahoo.com.ar, lorederosa@yahoo.com, adrianaelago@hotmail.com, lilianatraiber@gmail.com, patricio.nusshold@gmail.com, guillermina.rutsztein@gmail.com, ekeegan@psi.uba.ar

1 Proyecto de Investigación acreditado y financiado por la Universidad de Buenos Aires, Programación Científica 2011-2014 (UBACyT)

"Evaluación de una intervención psicoeducativa sobre perfeccionismo en estudiantes universitarios".
} 
profiles and their academic performance, it was found that students with an AP profile have a significantly higher average grades than those in the other two groups, and less pending exams. No significant differences were found in average grades between MP and NP. For the remaining variables, no significant differences were found between the different profiles of perfectionism. Implications of these findings for future research in the area are discussed.

Key words: perfectionism - academic performance - university students

\title{
PERFECCIONISMO E DESEMPENHO ACADÊMICO EM ESTUDANTES UNIVERSITÁRIOS DA CIDADE AUTÔNOMA DE BUENOS AIRES
}

\author{
Resumo
}

\begin{abstract}
O presente artigo tem como objetivo analisar a relação entre os perfis de perfeccionismo e o desempenho acadêmico em uma mostra de estudantes universitários da cidade de Buenos Aires. O desempenho foi operacionalizado através das variáveis média academica geral percebida, nota da última prova, quantidade de provas pendentes e anos cursados. Participaram na pesquisa 465 estudantes pertencentes aos cursos de Psicologia (52,2\%) Engenharia (26,2\%) e Medicina (21,6\%). A distribuição da população de estudantes nos diferentes perfis de perfeccionismo (adaptativo- PA, desadaptativo- PD e não perfeccionistasNP) apresentou-se de acordo com as previsões derivadas da teoria e dos estudos prévios feitos pela nossa equipe de pesquisa. Com respeito à relação entre os diferentes perfis e o desempenho acadêmico, encontrou-se que os estudantes com um perfil de PA apresentaram uma médica percebida significativamente superior, e uma quantidade de provas pendentes significativamente menor que os estudantes dos outros perfis. Não se evidenciaram diferenças significativas para estas variáveis entre os sujeitos com um perfil de PD e os estudantes NP. Para as restantes variáveis não foram encontradas diferenças significativas entre os diferentes perfis de perfeccionismo. Discutem-se as implicações destes descobrimentos para pesquisas futuras na área.

Palavras Chave: perfeccionismo, desempenho acadêmico, estudantes universitários.
\end{abstract}

\section{INTRODUCCIÓN}

La literatura científica ha conceptualizado el perfeccionismo como un rasgo negativo y unidimensional (Blatt, 1995; Hewitt, Flett, \& Ediger, 1996), principalmente a través de reportes clínicos. A finales de la década del noventa, los investigadores comenzaron a estudiar el fenómeno desde enfoques metodológicos más amplios, abandonando la perspectiva unidimensional por una multidimensional, que incluye tanto aspectos adaptativos como desadaptativos del mismo (Ashby, Kottman, \& Schoen, 1998; Hamacheck, 1978; Rice \& Slaney, 2002; Slaney, Mobley, Trippi, Ashby, \& Johnson, 1996).

Según el diccionario de la Real Academia Española, el perfeccionismo es definido como la tendencia a mejorar indefinidamente un trabajo sin decidirse a considerarlo acabado (RAE, 2001). Algunos autores que estudiaron este constructo, como Slaney y Ashby (1996), encontraron que cuando se les pedía a los perfeccionistas que se describieran y definieran el perfeccionismo, aludían principalmente a sus altos estándares personales. Estos últimos se corresponderían con los aspectos adaptativos de dicho rasgo, mientras que la discrepancia, que es la distancia percibida entre los propios estándares y el desempeño real obtenido, representaría el aspecto desadaptativo o negativo del perfeccionismo (Slaney, Rice, Mobley, Trippi, \& Ashby, 2001). De acuerdo con esta formulación, un perfil de per- feccionismo se caracteriza por la combinatoria de ambas variables: los perfeccionistas adaptativos presentarían altos estándares de desempeño y una baja discrepancia, a diferencia de los perfeccionistas desadaptativos, que presentarían altos estándares de desempeño y un alto nivel de discrepancia. En este sentido, diversos autores vincularon correlatos psicológicos negativos al perfil desadaptativo, y positivos al perfil adaptativo. Por ejemplo, los perfeccionistas adaptativos tendrían una autoestima más elevada y una mayor satisfacción con la vida que los perfeccionistas desadaptativos (Rice \& Dellwo, 2002).

\section{El perfeccionismo en estudiantes universitarios}

Algunos estudios han informado que un $70 \%$ de los estudiantes universitarios evaluados presentan rasgos perfeccionistas (Rice \& Slaney, 2002; Suddarth \& Slaney, 2001). Dichos rasgos se evidenciarían en este ámbito a través de comportamientos tales como la meticulosidad en el estudio y una excesiva preocupación por obtener altos niveles de rendimiento. También se manifiestan en la preocupación por no fracasar en términos académicos, así como por la postergación de las situaciones de examen (Blankstein, Dunkley, \& Wilson, 2008), y su correspondiente influencia en la autoeficacia académica (Yao, 2009).

Nuestro equipo se ha dedicado a investigar el perfeccionismo y su relación con el distrés psicológico y la calidad de vida en estudiantes universitarios. En coherencia con 
resultados previos (Rice \& Slaney, 2002; Suddarth et al., 2001), hemos hallado que más de la mitad de los estudiantes evaluados puntuaron como perfeccionistas de acuerdo con una medida multidimensional de dicho constructo, la APS-R, Almost Perfect Scale Revised (Slaney et al., 2001, adaptada por Arana, Keegan, \& Rutsztein, 2009). A su vez, se halló una correlación positiva entre perfeccionismo desadaptativo y malestar emocional (Arana et al., 2009).

\section{Perfeccionismo y desempeño académico}

El promedio académico general percibido (PAGP) ha sido utilizado como una medida de logro y desempeño académico en estudiantes universitarios por diversos investigadores. Burger (1992), por ejemplo, encontró que quienes tenían un alto deseo de control aspiraban a alcanzar promedios académicos superiores, $\mathrm{y}$ de hecho obtenían los promedios académicos más altos, en comparación con aquellos estudiantes con un bajo deseo de control. Siguiendo esta línea, los estudios que se centraron en el perfeccionismo utilizaron el PAGP de un estudiante como una variable para medir las diferencias entre los perfeccionistas y los no perfeccionistas (Rice \& Ashby, 2007; Rice \& Dellwo, 2002).

Algunas investigaciones citadas por Brumbaughy y colaboradores (2007) han sugerido que los estudiantes no perfeccionistas tienden a obtener promedios académicos menores que los perfeccionistas, tanto aquellos con un perfil adaptativo como los que presentan uno desadaptativo (Grzegorek, Slaney, Franze \& Rice, 2004; Rice \& Slaney, 2002; Rice \& Ashby, 2007). También se ha observado una diferencia significativa entre los promedios académicos de los perfeccionistas adaptativos y desadaptativos, siendo los primeros los que presentarían promedios mayores (Ashby \& Bruner, 2005; Rice \& Ashby, 2007). Así, algunos autores han señalado una correlación positiva entre los altos estándares y el desempeño académico, mientras que la discrepancia correlacionaría de manera negativa con el mismo (Leenards \& Lester, 2006).

No obstante, la investigación llevada a cabo por Brumbaugh y colaboradores (2007) evidenció resultados contradictorios con los anteriormente citados, concluyendo que no habría diferencias significativas en los promedios académicos de los dos grupos de perfeccionistas. Otro estudio (Grzegorek, et al., 2004) tampoco encontró tales diferencias entre los promedios y los perfiles de perfeccionismo, aunque se señaló que los estudiantes con altos valores de discrepancia expresaban estar más preocupados, menos satisfechos con sus logros y más descontentos en relación con su promedio académico general. Esto sugiere que los estudiantes perfeccionistas desadaptativos serían particularmente autocríticos. Por el contrario, los no perfeccionistas muestran puntuaciones más bajas en medidas de evaluación de las normas personales (altos estándares), la autocrítica, la duda de sí mismos, y en la preocupación por cometer errores (Rice \& Ashby, 2007).

Por otra parte, se ha señalado que el perfeccionismo desadaptativo podría acarrear un impacto negativo en el rendimiento académico, asociándose con diversos trastornos y contribuyendo al desgaste psicológico e incluso al abandono o postergación de metas (Wang, Slaney, \& Rice, 2007; Hewitt \& Flett, 1991; Szabo \& Terre-Blanche, 1997; Ashby et al., 1998). Desde esta perspectiva, algunos autores (Martínez Martínez, Bresó Esteve, Llorens Gumbau \& Grau Gumbau, 2005) identificaron elementos que en los estudiantes operarían como obstáculos o como facilitadores en el cumplimiento de tareas académicas. Martínez Martínez y colaboradores (2005) hallaron una relación positiva entre obstáculos en el estudio, burnout y propensión al abandono. En este sentido, Freudenberger (1974) sugirió que era más probable que el síndrome de burnout se presentase en los perfeccionistas, ya que la dificultad para alcanzar los altos niveles de rendimiento esperados incrementaría la angustia, conduciendo a una mayor fatiga (Magnusson, Nias \& White, 1996). Los elementos facilitadores, en cambio, se relacionarían positivamente con el compromiso, la autoeficacia y la satisfacción relacionadas con el estudio. Como evidencian Zhang y colaboradores (2005), las puntuaciones de los estudiantes perfeccionistas adaptativos correlacionarían positivamente con aspectos vinculados con el compromiso con dicha actividad.

Otros autores (Yeong-giKyeon, Sung-myeongCho, Hwyeon-gukHwang \& Kang-Uk Lee, 2010) también han destacado los efectos mediadores del perfeccionismo sobre el rendimiento académico, los problemas emocionales como la depresión y el agotamiento psicológico o burnout.

\section{Objetivos e hipótesis}

El presente estudio es de naturaleza comparativo-correlacional y se centra en determinar si existe una asociación estadísticamente significativa entre perfiles de perfeccionismo y el desempeño académico, dimensión operacionalizada a través de las siguientes variables: promedio académico general percibido (PAGP), calificación del último examen (CUE), cantidad de exámenes adeudados (EA), y años de carrera cursados (AC).

Nuestra hipótesis postula, a partir de los estudios desarrollados hasta el presente, que el rendimiento académico diferirá en función del estilo de perfeccionismo que posea cada estudiante. En particular, esperamos que los sujetos no perfeccionistas informen rendimientos académicos inferiores que aquellos individuos con perfiles perfeccionistas, tanto adaptativos como desadaptativos. A su vez, se espera que 
los perfeccionistas desadaptativos tiendan a adeudar mayor cantidad de exámenes y que demoren mayor cantidad de años en cursar la carrera.

\section{MÉTODO}

\section{Participantes}

En este estudio se incluyeron estudiantes universitarios cuya edad estuviese comprendida en el rango de 18 a 30 años. La participación fue voluntaria, incluyéndose sujetos de ambos sexos. 465 estudiantes, pertenecientes a tres unidades académicas, cumplieron los criterios de inclusión y aceptaron participar voluntariamente en la investigación. La distribución por carrera fue la siguiente: 241 estudiantes de Psicología (52,2\%), 100 estudiantes de Medicina (21,6\%); ambas carreras de grado pertenecientes a la Universidad de Buenos Aires; 121 estudiantes de Ingeniería (26,2\%) de la Universidad Tecnológica Nacional y tres casos donde falta la información. La edad media fue de 23,49 años (DE $2,59)$ y la distribución por género fue de $63,2 \%(\mathrm{~N}=294)$ mujeres y $36,8 \%(\mathrm{~N}=171)$ varones.

\section{Instrumentos}

-APS-R. Almost Perfect Scale Revised (Slaney et. al, 2001; adaptación: Arana et al., 2009). Esta escala está compuesta por 23 reactivos distribuidos en tres subescalas: Altos Estándares, Orden y Discrepancia. Presenta formato de respuesta tipo Likert con siete opciones, indicando grado de acuerdo con las afirmaciones. Este instrumento permite discriminar perfiles desadaptativos o disfuncionales y perfiles adaptativos o funcionales de perfeccionismo.

\section{Procedimiento}

El protocolo fue administrado a los estudiantes en las distintas unidades académicas, durante sus horas de clase, con el consentimiento de sus docentes y de las autoridades de las respectivas universidades. Se distribuyeron los protocolos, se contestaron las inquietudes que surgieron al momento de completarlos, y se procedió a obtener el consentimiento informado de los participantes, en donde se garantizó la confidencialidad de la información recolectada, así como la posibilidad de abandonar su participación en cualquier instancia del estudio.

Se solicitó a los estudiantes referir datos socio-demográficos tales como edad, nacionalidad, género y estado civil. También informaron datos relativos a aspectos académicos: universidad, carrera en curso, año de ingreso a la carrera, promedio académico general percibido, calificación del último examen y cantidad de exámenes adeudados.

Los perfiles de perfeccionismo fueron establecidos a través de agrupaciones por punto de corte (Kottman, T. \& Ashby, J., 1999) a partir de las subescalas de la APS-R (Slaney et al., 2001; adaptación Arana et al., 2009). De esta manera, todos los estudiantes resultaron clasificados en uno de los perfiles de perfeccionismo: no perfeccionista (NP), perfeccionista adaptativo (PA), y perfeccionista desadaptativo (PD). Por otra parte, la variable desempeño académico fue operacionalizada a través de cuatro medidas: promedio académico general percibido (PAGP), calificación del último examen (CUE), cantidad de exámenes adeudados (EA) y años de carrera cursados (AC).

\section{RESULTADOS}

La proporción de perfiles de perfeccionismo (NP, PA y PD) concuerda con lo esperado, ajustándose a la teoría y las publicaciones anteriores de nuestro equipo de investigación (Arana et al., 2010; Arana et al., 2009). Estos resultados se detallan en la tabla 1. Para comparar los grupos, se utilizó un análisis multivariado de la varianza (MANOVA) indicando como factor los perfiles de perfeccionismo (NP, PA y PD), y las medidas de desempeño como variables dependientes: promedio académico general percibido (PAGP), calificación del último examen (CUE), cantidad de exámenes adeudados (EA), y años de carrera cursados (AC). El efecto multivariado fue estadísticamente significativo, $\Lambda$ de Wilks $=, 910 ; F(8 ; 696)=4,2 ; p<, 05 ; \eta^{2 \text { parcial }}=, 046$; potencia observada .99 . Este análisis reveló diferencias estadística-

Tabla 1

Perfiles y dimensiones de perfeccionismo

\begin{tabular}{lccccc}
\hline \multirow{2}{*}{ APS-R } & $\mathbf{N P}(\mathbf{n}=\mathbf{2 7 4})$ & $\mathbf{P A}(\mathbf{n}=\mathbf{8 6})$ & $\mathbf{P D}(\mathbf{n}=\mathbf{1 0 0})$ & $\mathbf{F}$ & $\mathbf{g l}$ \\
\cline { 2 - 4 } & $\mathbf{M}(D E)$ & $\mathbf{M}(D E)$ & $\mathbf{M}(D E)$ & & \\
\hline Altos Estándares & $33,12(4,10)_{\mathrm{a}}$ & $41,30(2,16)_{\mathrm{b}}$ & $42,34(2,62)_{\mathrm{c}}$ & 342,71 & $2 ; 457$ \\
\hline Discrepancia & $38,59(12,7)_{\mathrm{a}}$ & $29,1(4,9)_{\mathrm{b}}$ & $51,27(11,1)_{\mathrm{c}}$ & 93,1 & $2 ; 448$ \\
\hline
\end{tabular}

* 5 casos perdidos

$* *$ los valores medios de $F$ resultaron significativos al $p<.0001$ 
Tabla 2

Variables de desempeño. Comparación entre perfiles de perfeccionismo.

\begin{tabular}{cccc}
\hline \multirow{2}{*}{ Desempeño Académico } & $\mathbf{N P}(\mathbf{n}=\mathbf{2 7 4})$ & $\mathbf{P A}(\mathbf{n}=\mathbf{8 6})$ & PD $(\mathbf{n}=\mathbf{1 0 0})$ \\
\cline { 2 - 4 } & $\mathbf{M}(\mathbf{D E})$ & $\mathbf{M}(\mathbf{D E})$ & $\mathbf{M}(\mathbf{D E})$ \\
\hline PAGP & $7,08(, 06)_{\mathrm{b}}$ & $7,69(, 12)_{\mathrm{a}}$ & $7,34(, 09)_{\mathrm{b}}$ \\
CUE & $6,99(, 12)$ & $7,45(, 24)$ & $7,02(, 20)$ \\
EA & $1,15(, 82)_{\mathrm{b}}$ &, $64(, 16)_{\mathrm{a}}$ & $1,11(, 14)_{\mathrm{b}}$ \\
AC & $5,02(, 152)$ & $4,57(, 30)$ & $4,22(, 251)$ \\
\hline
\end{tabular}

** los valores medios denotados con subíndices son significativos al $p<.05$

mente significativas entre perfiles para PAGP y EA, mas no así para CUA y AC. PAGP: $[F(2,351)=11,81 ; p<, 0001$; $\left.\eta^{2 \text { parcial }}=, 063\right]$ y para EA: $[F(2,351)=3,754 ; p<, 024$; $\left.\eta^{2 \text { parcial }}=, 021\right]$. En particular, se halló que los estudiantes con un perfil perfeccionista adaptativo percibían su promedio académico significativamente mejor que los estudiantes no perfeccionistas y los estudiantes con un perfil de PD. No se evidenciaron diferencias significativas para esta variable entre esos dos últimos grupos (NP y PD). Se encontró, además, una diferencia entre perfiles para la cantidad de exámenes adeudados. Los estudiantes con un perfil de perfeccionismo adaptativo difirieron de manera significativa de aquellos con un perfil no perfeccionista y perfeccionista desadaptativo, presentando una menor cantidad de exámenes adeudados. No se evidenciaron diferencias significativas para esta variable entre estos perfiles (NP y PD).

Estos resultados, en conjunto con las comparaciones post-hoc, se presentan en la tabla 2.

\section{DISCUSIÓN}

El objetivo del presente estudio fue determinar si existía una asociación estadísticamente significativa entre los perfiles de perfeccionismo y algunas variables ligadas al desempeño académico en una muestra de estudiantes universitarios. De acuerdo con lo esperado, se halló que los estudiantes con un perfil de perfeccionismo adaptativo (PA) obtuvieron las puntuaciones más elevadas en la variable promedio académico general percibido (PAGP), al mismo tiempo que evidenciaron una menor cantidad de exámenes adeudados. Se diferenciaron significativamente de los estudiantes no perfeccionistas (NP) y de los estudiantes con un perfil de perfeccionismo desadaptativo (PD). No obstante, no se hallaron diferencias estadísticamente significativas para estas variables entre los estudiantes con un perfil de PD y los estudiantes NP.
De manera, congruente con estos resultados, algunos autores han señalado una correlación positiva entre los altos estándares y el desempeño académico, mientras que la discrepancia correlacionaría de manera negativa con el mismo (Leenards \& Lester, 2006). Asimismo, otros estudios han mostrado una diferencia significativa entre los promedios académicos de PA y PD, siendo los primeros los que presentaban mejores puntuaciones (Ashby \& Bruner, 2005; Rice \& Ashby, 2007). En nuestro estudio los sujetos con un perfil de PD informaron un PAGP significativamente más bajo que los PA. En este sentido, se ha señalado que el PD podría implicar no sólo un impacto negativo en el desempeño académico, sino que también se asociaría con diversos trastornos, contribuyendo al desgaste psicológico e incluso al abandono o postergación de metas (Wang et al., 2007; Hewitt \& Flett, 1991; Szabo \& Terre-Blanche, 1997; Ashby et al., 1998). Sin embargo, dicha asociación podría no ser tan lineal a la luz de ciertos hallazgos. Svanum \& Zody (2001), por ejemplo, revelaron que estudiantes con algún tipo de trastorno de ansiedad presentaron promedios académicos realmente elevados. Una fuerte necesidad de control, esperable en este perfil de estudiantes, podría incidir de manera positiva en su desempeño académico, aunque a costa de un gran malestar.

Por otra parte, los estudiantes con un perfil de PA difirieron de los NP y de los estudiantes con un perfil de PD en tener una menor cantidad de exámenes pendientes. Se evidenciaría en este sentido un aspecto de las implicaciones positivas del perfeccionismo en su dimensión adaptativa en la población de estudiantes universitarios. Asimismo, estos hallazgos nos permitirían pensar una posible implicación del PD: la procrastinación. Definida como una tendencia a posponer la culminación de una tarea que usualmente resulta en un estado de insatisfacción o malestar subjetivo (Burka \& Yuen, 1983), la procrastinación en estudiantes se vincularía con el miedo al fracaso (Solomon \& Rothblum, 
1984). Esto resulta congruente si consideramos que los estudiantes con un perfil de PD suelen presentar autoevaluaciones excesivamente críticas, asociadas con altos estándares de desempeño y altos niveles de discrepancia.

Cabe destacar también que para las dos variables anteriormente mencionadas (PAGP y EA) no se evidenciaron diferencias significativas entre los perfiles PD y NP; ambos perfiles evidenciaron un PAGP significativamente más bajo y adeudar más exámenes que los PA. Esto sugiere que a pesar de que los PD tienen estándares más altos que los NP, no lograrían alcanzar un rendimiento congruente con los mismos. En este sentido, para esta muestra se evidenció el impacto del perfeccionismo en su dimensión desadaptativa sobre el desempeño académico de los estudiantes.

En relación con la cantidad de exámenes adeudados, cabe señalar que en algunas unidades académicas, como la Facultad de Psicología, existe una normativa que limita la cantidad de exámenes que un alumno puede adeudar para mantener su regularidad. Esto representa una limitación del presente estudio, pudiendo atenuar las diferencias para esta variable.

Cabe citar otra limitación inherente al presente estudio, vinculada con la variable ligada al promedio. En la presente muestra se utilizó el promedio percibido que reportaron los propios estudiantes como una de las medidas de desempeño académico. La tendencia a la autocrítica inherente al PD podría estar sesgando la percepción de esta variable en los estudiantes con dicho perfil. En este sentido, sería interesante poder contrastar los resultados obtenidos en el presente trabajo con futuros estudios que contemplen otras medidas de la variable ligada al promedio en nuestra población.

De manera contraria a lo esperado, no se hallaron diferencias entre los perfiles de perfeccionismo para la variable calificación del último examen (CUA). Esto podría deberse a que, a diferencia del PAGP, la CUA sería un indicador de desempeño más situacional, que no necesariamente se asociaría de manera directa con un desempeño académico global. Por consiguiente, y de acuerdo al menos con los resultados obtenidos en este estudio, dicha variable no resultaría adecuada para el tipo de análisis que se busca realizar desde esta perspectiva.

Tampoco se hallaron diferencias entre perfiles para la variable cantidad de años de carrera cursados (AC). Si bien la misma podría resultar un indicador indirecto de la tendencia de los estudiantes perfeccionistas, en particular los PD, a la procrastinación, resta evaluarlo en futuras investigaciones en el área. Por otra parte, cabe destacar que los criterios de inclusión por rango etario, favorecen la homogeneidad de la muestra pero al mismo tiempo se constituyen en una limitación del presente estudio. Dichos criterios podrían estar sesgando los resultados para esta variable (AC), al no haberse incluido sujetos de mayor edad, que posterguen metas académicas sistemáticamente y lleven más años realizando sus estudios de grado.

Finalmente, futuros estudios podrán, asimismo, dar cuenta del papel de los aspectos anteriormente mencionados en la relación entre el perfeccionismo y el desempeño académico de los estudiantes en diversos contextos, teniendo en cuenta otras variables no contempladas en el presente trabajo, tales como el tipo de carrera y las estrategias de afrontamiento implementadas por los estudiantes ante las exigencias académicas.

\section{REFERENCIAS}

Arana, F., Scappatura, M. L., Miracco, M., Elizathe, L., Rutsztein, G. \& Keegan, E. (2010). Perfeccionismo positivo / negativo y percepción de calidad de vida en estudiantes de Psicología, Medicina e Ingeniería de la Ciudad de Buenos Aires. Anuario de Investigaciones de la Facultad de Psicología de la Universidad de Buenos Aires, 17.

Arana, F., Keegan, E. \& Rutsztein, G. (2009). Adaptación de una medida multidimensional de perfeccionismo: la Almost Perfect Scale-Revised (APS-R). Un estudio preliminar sobre sus propiedades psicométricas en una muestra de estudiantes argentinos. Revista Evaluar Publicación Anual del Laboratorio de Evaluación Psicológica y Educativa de la Facultad de Psicología de la Universidad Nacional de Córdoba, 9, 35-53.

Arana, F., Scappatura, M. L., Miracco, M., Elizathe, L., Rutsztein, G. \& Keegan, E. (2009). Un estudio sobre perfeccionismo en estudiantes universitarios argentinos: resultados preliminares en estudiantes de Psicología. Anuario de Investigaciones de la Facultad de Psicología de la Universidad de Buenos Aires, 14(1), 17-24.

Ashby, J.S., Kottman, T. \& Schoen, E. (1998). Multidimensional perfectionism and eating disorders. Journal of Mental Health Consulting, 20, 261-271.

Ashby, J. S,. \& Bruner, L. P. (2005). Multidimensional perfectionism and obsessive-compulsive behaviors. Journal of College Counseling, 8(1), 31-40.

Blankstein, K. R., Dunkley, D. M. \& Wilson, J. (2008). Concerns and Personal Standards Perfectionism: Self-esteem as a Mediator and Moderator of Relations with Personal and Academic Needs and Estimated GPA. Current Psychology, 27, 29-61.

Blatt, S. J. (1995). The destructiveness of perfectionism: Implications for the treatment of depression. American Psychologist, 50, 1003-1020.

Burger, J. M. (1992). Desire for control and academic performance. Canadian Journal of Behavioural Science, 24(2), 147-155. 
Brumbaugh, J., Lepsik, R. \& Olinger, Ch. (2007). The Relationship between GPA and Perfectionism. Undergraduate Research Journal for the Human Sciences, 6, 147-155.

Burka, J. B. \& Yuen, L. M. (1983). Procrastination: Why you do it and what to do about it. Reading, PA: Addison-Wesley.

Freudenberger, H. J. (1974). Staff burnout. Journal of Social Issues, 30(1), 159-165.

Frost, R. O., Heimberg, R. G., Halt, C. S., Mattia, J. L. \& Neubauer, A. L. (1993). A comparison of two measures of perfectionism. Personality and Individual Differences, 14, 99- 126.

Grzegorek, J. L., Slaney, R. B., Franze, S. \& Rice, K. G. (2004). Self-criticism, dependency, self-esteem, and grade point average satisfaction among clusters of perfectionists and non perfectionists. Journal of Counseling Psychology, 51(2), 192-200.

Hamachek, D. E. (1978). Psychodynamics of normal and neurotic perfectionism. Psychology, 15, 27-33.

Hewitt, P. L. \& Flett, G. L. (1991). Perfectionism in the self and social contexts: Conceptualization, assessment, and association with psychopathology. Journal of Personality and Social Psychology, 60, 456-470.

Hewitt, P. L., Flett, G. L, \& Ediger, E. (1996). Perfectionism and depression: Longitudinal assessment of a specific vulnerability hypotheses. Journal of Abnormal Psychology, 105, 276-280.

Kottman, T. \& Ashby, J. (1999). Social interest and multidimensional perfectionism. Journal of Individual Psychology, $55,176-185$.

Leenards, L. \& Lester, D. (2006). Perfectionism, Depression, and Academic Performance. Psychological Reports, 99, 941-942.

Magnusson, A. E., Nias, D. K. \& White, P. D. (1996). Is perfectionism associated with fatigue? Journal of Psychosomatic Research, 41, 377-383.

Martínez Martínez, I., Bresó Esteve, E., Llorens Gumbau, S. \& Grau Gumbau, R. (2005). Bienestar psicológico en estudiantes universitarios: facilitadores y obstaculizadores del desempeño académico. Anales de Psicología de la Universidad de Murcia, 21(1), 170-180.

McKenzie, K., Gow, K, \& Schweitzer, R. (2004). Exploring first-year academic achievement through structural equation modelling. Higher Education Research \& Development, 23(1), 95-112.

Nguyen, N. T., Allen, L. C. \& Fraccastoro, K. (2005). Personality predicts academic performance: Exploring the moderating role of gender. Journal of Higher Education Policy \& Management, 27(1), 105-116.

Nounopoulos, A., Ashby, J. \& Gilman, R. (2006). Coping resources, perfectionism, and academic performance among adolescents. Psychology in the Schools, 43, 613-622.

Real Academia Española (2001). Diccionario de la Lengua Española (22da. ed.).Consultado en http://lema.rae.es/ drae/?val=perfeccionismo
Rice, K. \& Slaney, R. B. (2002). Clusters of Perfectionistics: Two Studies of Emotional Adjustment and Academic Achievement. Measurement \& Evaluation in Counseling \& Development, 35-48.

Rice, K. G. \& Dellwo, J. P. (2002). Perfectionism and self-development: Implications for college adjustment. Journal of Counseling \& Development, 80(2), 188.

Rice, K. G. y Ashby, J. S. (2007). An efficient method for classifying perfectionists. Journal of Counseling Psychology, 54(1), 72-85.

Slaney, R. B., Mobley, M., Trippi, J., Ashby, J. S. \& Johnson, D. (1996). Almost Perfect Scale-Revised. Unpublished scale, The Pennsylvania State University, University Park.

Slaney, R. B. \& Ashby, J. S. (1996). Perfectionists: Study of a criterion group. Journal of Counseling and Development, 74, 393-398.

Slaney, R. B., Rice, K. G. Mobley, M. Trippi, J. \& Ashby, J. S. (2001). The revised Almost Perfect Scale. Measurement and Evaluation in Counseling and Development, 34, 130145.

Speirs Neumeister, K. (2004). Understanding the relationship between perfectionism and achievement motivation in gifted college students. Gifted Child Quarterly, 48(3), 219-231.

Solomon, L. J. \& Rothblum, E. D. (1984). Academic procrastination: Frequency and cognitive-behavioral correlates. Journal of Counseling Psychology, 31, 503-509.

Suddarth, B. H. \& Slaney, R. B. (2001). An investigation of the dimensions of perfectionism in college students. Measurement and Evaluation in Counselling and Development, 34(3), 157-165.

Svanum, S. \& Zody, Z. B. (2001). Psychopathology and college grades. Journal of Counseling Psychology, 48(1), 72-76.

Szabo, C. P. \& Terre Blanche, M. J. (1997). Perfectionism in anorexia nervosa. American Journal of Psychiatry, 154, 132.

Wang, K. T., Slaney, R. B. \& Rice, K. G. (2007). Perfectionism in Chinese university students from Taiwan: A study of psychological well-being and achievement motivation. Personality and Individual Differences, 42, 1279-1290.

Yao, M. (2009). An exploration of multidimensional perfectionism, academic self-efficacy, procrastination frequency, and Asian American cultural values in Asian American university students. Disertación doctoral no publicada, Ohio State University, Ohio. EE. UU.

Yeong-giKyeon, Sung-myeong Cho, Hwyeon-guk Hwang \& Kang-Uk Lee (2010). The Effects of Perfectionism on Academic Achievement in Medical Students. The Korean Society of Medical Education, 22(3), 205-214.

Zhang,Y., Gan,Y. \& Cham, H. (2005). Perfectionism, academic burnout and engagement among Chinese college students: A structural equation modelling analysis. Personality and Individual Differences, 43, 1529-1540. 